\title{
Correction: Development of an Online Health Care Assessment for Preventive Medicine: A Machine Learning Approach
}

Cheng-Sheng Yu ${ }^{1,2}, \mathrm{PhD}$; Yu-Jiun Lin ${ }^{1,2}$, MD; Chang-Hsien Lin ${ }^{1,2}, \mathrm{MD}$; Shiyng-Yu Lin ${ }^{1,2}, \mathrm{MD}$; Jenny L Wu ${ }^{1,2}$, BSc; Shy-Shin Chang ${ }^{1,2}$, MD, PhD

${ }^{1}$ Department of Family Medicine, Taipei Medical University Hospital, Taipei, Taiwan
${ }^{2}$ Department of Family Medicine, School of Medicine, College of Medicine, Taipei Medical University, Taipei, Taiwan

Corresponding Author:

Shy-Shin Chang, MD, PhD

Department of Family Medicine

School of Medicine

College of Medicine, Taipei Medical University

250 Wuxing St

Taipei, 11031

Taiwan

Phone: 886223565926

Email: sschang0529@gmail.com

\section{Related Article:}

Correction of: https://www.jmir.org/2020/6/e18585/

(J Med Internet Res 2020;22(7):e21753) doi: 10.2196/21753

In "Development of an Online Health Care Assessment for Preventive Medicine: A Machine Learning Approach" (J Med Internet Res 2020;22(6):e18585) the authors noticed an error in the Institutional Review Board (IRB) approval number.

Under the Ethics heading in the Methods section, the IRB approval number was listed as "N201906023"

(TMUH TMU-JIRB number N201906023)
The correct number is "N202003088":

(TMUH TMU-JIRB number N202003088)

The correction will appear in the online version of the paper on the JMIR Publications website on July 27, 2020, together with the publication of this correction notice. Because this was made after submission to PubMed, PubMed Central, and other full-text repositories, the corrected article has also been resubmitted to those repositories.

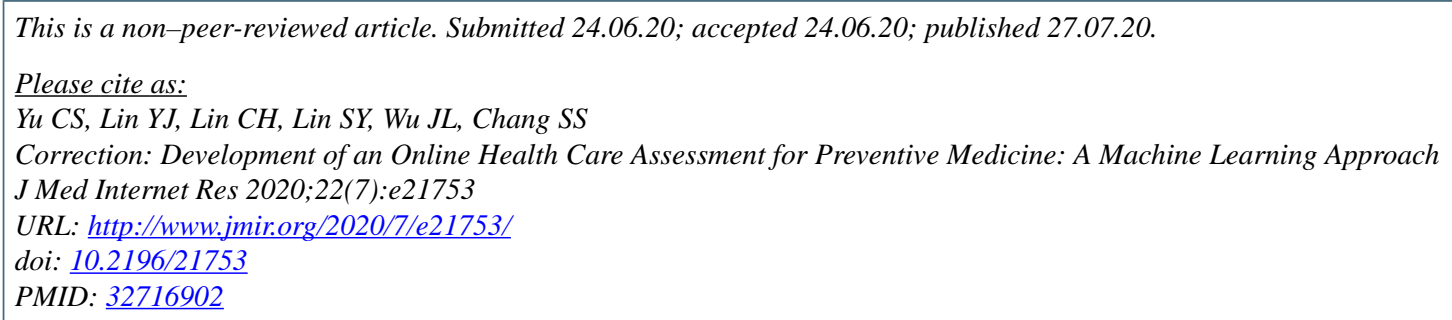

(C) Cheng-Sheng Yu, Yu-Jiun Lin, Chang-Hsien Lin, Shiyng-Yu Lin, Jenny L Wu, Shy-Shin Chang. Originally published in the Journal of Medical Internet Research (http://www.jmir.org), 27.07.2020. This is an open-access article distributed under the terms of the Creative Commons Attribution License (https://creativecommons.org/licenses/by/4.0/), which permits unrestricted use, distribution, and reproduction in any medium, provided the original work, first published in the Journal of Medical Internet Research, is properly cited. The complete bibliographic information, a link to the original publication on http://www.jmir.org/, as well as this copyright and license information must be included. 